\title{
Long-lived plasma cells are generated in mucosal immune responses and contribute to the bone marrow plasma cell pool in mice
}

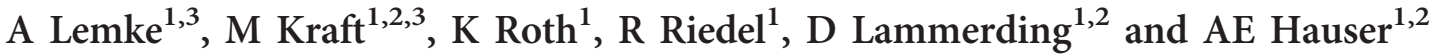

During systemic immune responses, plasma blasts are generated in secondary lymphoid organs and migrate to the bone marrow, where they can become long-lived, being responsible for the maintenance of long-term antibody titers. Plasma blasts generated in mucosal immune responses of the small intestine home to the lamina propria (LP), producing mainly immunoglobulin A. The migration of these antibody-secreting cells is well characterized during acute immune responses. Less is known about their lifetime and contribution to the long-lived bone marrow compartment. Here we investigate the lifetime of plasma cells (PCs) and the relationship between the PC compartments of the gut and bone marrow after oral immunization. Our findings indicate that PCs in the LP can survive for extended time periods. PCs specific for orally administered antigens can be detected in the bone marrow for at least 9 months after immunization, indicating that the mucosal PC compartment can contribute to the long-lived PC pool in this organ, independent of the participation of splenic B cells. Our findings suggest that the compartmentalization between mucosal and systemic PC pools is less strict than previously thought. This may have implications for the development of vaccines as well as for autoantibody-mediated diseases.

\section{INTRODUCTION}

As the producers of soluble immunoglobulins (Igs), plasma cells (PCs) are crucial effector cells of humoral immunity. In systemic immune responses, antigen-specific plasma blasts are generated from activated B cells in secondary lymphoid organs. A fraction of those motile plasma blasts leave the secondary lymphoid organs and migrate via the blood to the bone marrow. ${ }^{1,2}$ Here plasma blasts that encounter a special microenvironment termed the survival niche can become long-lived PC. PC survival requires the combination of certain molecular signals that act synergistically ${ }^{3}$ and are derived from various cellular sources: the tumor necrosis factor-family cytokine A proliferation-inducing ligand (APRIL) is mainly provided by eosinophils, ${ }^{4}$ whereas the CXC chemokine ligand 12 (CXCL12) is derived from resident stromal cells in the bone marrow. ${ }^{5}$ CXCL12, also known as stromal cell derived factor $1 \alpha$, acts by attracting migratory plasma blasts to the bone marrow as well as by supporting the survival of resident, sessile PCs. ${ }^{6}$ In addition to those soluble factors, adhesion molecules such as very late antigen 4 and lymphocyte function-associated antigen 1 contribute to the retention of PCs in their niches by binding to vascular cell adhesion molecule 1 and fibronectin, respectively, on the surface of stromal cells. ${ }^{7}$

The majority of plasma blasts in the blood of humans in steady state are derived from mucosal immune responses. ${ }^{8} \mathrm{~A}$ large fraction of antibody-secreting cells (ASCs) in the body are located in the lamina propria (LP), and the vast majority of LP PCs produce antibodies of the immunoglobulin A (IgA) isotype. Gut-associated lymphoid tissue provides an environment rich in factors such as transforming growth factor beta and APRIL, which promote class switching of B cells to IgA. ${ }^{9}$ Mucosal IgA ${ }^{+}$plasma blasts first migrate through lymphatic vessels to the mesenteric lymph nodes, and subsequently they enter the blood circulation through the thoracic duct and home back to the LP. The CC chemokine receptor 9 (CCR9) and CCR10 on plasma blasts are responsible for homing to the small

\footnotetext{
${ }_{1}^{1}$ Deutsches Rheuma Forschungszentrum (DRFZ), a Leibniz Institute, Berlin, Germany and ${ }^{2}$ Charité Universitätsmedizin, Charitéplatz 1, Berlin, Germany. Correspondence: AE Hauser (hauser@drfz.de)

${ }^{3}$ These authors contributed equally to this work. 
and large intestine, respectively. Retinoic acid has a role in imprinting of the gut-homing mechanism of $\mathrm{IgA}^{+}$plasma blasts by inducing CCR9. ${ }^{10}$ The adhesion molecule integrin $\alpha_{4} \beta_{7}$ also mediates gut tropism in plasma blasts. ${ }^{11}$ It has also been proposed that a fraction of IgA ${ }^{+}$PCs in the diffuse LP are generated locally, without the need for recirculation. ${ }^{12}$ By flow cytometry analysis, up to $50 \%$ of bone marrow PCs in mice have been shown to be $\operatorname{IgA}^{+}$. Although $\operatorname{IgA}^{+}$PCs evidently express CXC chemokine receptor 4 (CXCR4) and migrate to the bone marrow, ${ }^{13}$ it is not known to what extent they can contribute to the resident long-lived PC compartment. To this date, the information about the lifespan of intestinal PCs is sparse. Previous studies suggest that the vast majority of ASCs in the LP are short-lived, ${ }^{14,15}$ but there is indirect evidence in the recent literature that there might be a long-lived compartment present in this tissue as well, ${ }^{16,17}$ similar to the bone marrow. ${ }^{18}$ In general, systemic and mucosal antibody responses are thought to be compartmentalized, and the production of antibodies against oral antigens is therefore restricted to the gut. Here we investigate the lifetime of ASCs induced after oral immunization of mice in the LP of the small intestine and bone marrow and analyze the relationship between the intestinal and the bone marrow PC compartments.

\section{RESULTS}

\section{Antigen-specific PCs are present in the LP and bone} marrow for at least $\mathbf{3}$ months after oral immunization

To monitor the presence of antigen-specific PCs in the LP over time and analyze the relationship between PC compartments of the gut and bone marrow, we immunized mice orally with cholera toxin (CT). The oral administration of CT has been shown previously to elicit a strong T-cell-dependent PC response in the LP. ${ }^{19}$ After neutralization of gastric acid using sodium bicarbonate, mice were immunized via oral gavage with $10 \mu \mathrm{g}$ of $\mathrm{CT}$ and 3 weeks later the mice received a boost immunization (Figure 1a). Six days after boost, at a time point when the PC response was predicted to peak at the induction site based on kinetics generated in systemic immune responses, ${ }^{1}$ one cohort of mice was killed and the LP of the small intestine was analyzed by ELISPOT for the presence of CT-specific PCs. Non-immunized mice served as controls (Figure 1b). As expected, CT-specific IgA-ASCs were not detected at all in naive mice. In contrast, on average $>10^{5}$ specific IgA ${ }^{+}$ASCs were detected in the LP at day 6 after boost immunization. We also detected CT-specific ASCs of other isotypes, namely IgG and IgM at that time point; however, their total numbers were $\sim 10$ times lower than the number of IgAASCs. Ninety days after immunization, we could still detect $\sim 2.9 \times 10^{3}$ CT-specific IgA-ASCs in the LP, whereas IgG- and IgM-ASCs specific for the antigen, in contrast, were undetectable. To compare the persistence of CT-specific ASCs in the LP with those in other lymphoid tissues, we performed ELISPOT analyses of the bone marrow. At day 6 after oral boost, an average of $2.6 \times 10^{4}$ total CT-specific IgA-ASCs and $2.3 \times 10^{4}$ of specific IgG-ASCs were present in the bone marrow (Figure 1c). Although we noticed a reduction of each of the two isotypes after day 6, similar to previously published data on ASC accumulation in the bone marrow, ${ }^{1}$ we could still detect $7.7 \times 10^{3}$ specific IgG-ASCs (1/3 of the value at day 6$)$ and $5 \times 10^{3}$ (1/5 of the value at day 6) IgA-ASCs 90 days after boost. CT-specific IgM-ASCs were also present in the bone marrow, albeit in lower numbers as the other isotypes tested (on average: $6.7 \times 10^{3}$ and $6.4 \times 10^{2} 6$ and 90 days after oral boost, respectively).

\section{Oral administration of $\mathrm{CT}$ with co-administered ovalbumin (OVA) induces long-term persistence of CT- and OVA- specific PCs in the LP as well as the bone marrow}

$\mathrm{CT}$ has been described to function as a potent adjuvant for mucosal immune reactions. ${ }^{20}$ Furthermore, its adjuvant functions have been shown to promote long-term immunological memory in the gut to other immunogens that are applied orally together at the same time as $\mathrm{CT}^{21}$ This led us to test whether CT could promote long-term humoral immunity in the LP against co-administered antigens. Indeed, when we gavaged mice with a mix of CT and OVA we could detect similar numbers of IgA-secreting cells by ELISPOT against both antigens in the LP even at late time points, 9 months after immunization (Figure 2a, left panel). At the same time, we could also detect CT-specific as well as OVA-specific IgA-ASCs in the bone marrow at numbers similar to the ones present in the LP (Figure 2a, right panel). In contrast, both CT- and OVAspecific IgG-secreting ASCs were present in the bone marrow. To further confirm the data generated by ELISPOT, we also monitored the numbers of OVA-specific ASCs in the LP at different time points after boost by flow cytometry. OVAspecific ASCs were identified by staining intracellularly against the Ig kappa light chain in combination with fluorescently labeled OVA (Figure 2b). We confirmed that virtually all OVA-specific PCs in the LP and in the bone marrow were expressing the kappa light chain (see Supplementary Figure S1 online). Using this strategy, we were able to detect OVAspecific PCs in the LP up to 9 months after boost immunization (Figure $\mathbf{2 b}, \mathbf{c}$ ), thereby confirming the data generated by ELISPOT assay.

\section{ASCs induced after oral immunization can become long- lived and persist in the LP}

The antigen-specific ASCs that we detected at 9 months after immunization might represent long-lived PCs. Alternatively, they could be short-lived plasma blasts generated continuously by ongoing immune responses in the gut. To test this, we immunized mice with OVA and CT and fed them during the first 12 days of the boost response with the thymidine analog 5-ethynyl-2'-deoxyuridine (EdU) (Figure 3a), which gets stably incorporated in the DNA of proliferating cells. ${ }^{22}$ Proliferating plasma blasts in the LP were efficiently labeled with EdU: at day 12, directly after termination of the EdU feeding, $>90 \%$ of OVA-specific plasma blasts had incorporated EdU (Figure 3b). The nuclear localization of EdU in plasma blasts was confirmed by immunofluorescence histology (Figure 3c). Microscopic examination also clearly showed EdU uptake by OVA-specific PCs in the LP (Figure 3d). 
a

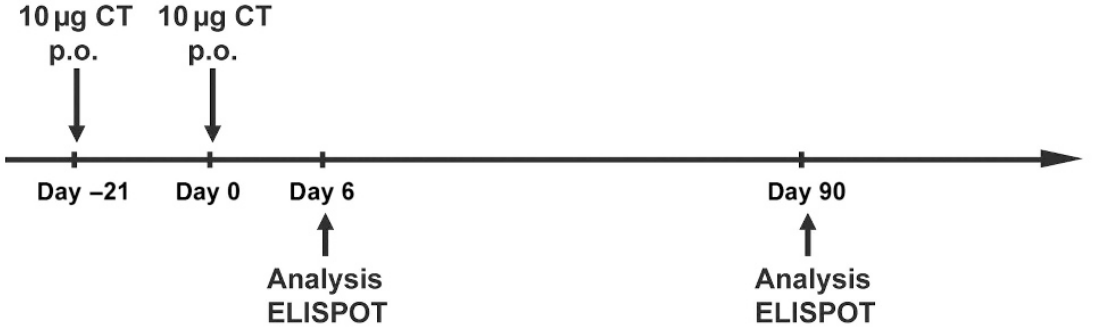

b

Lamina propria
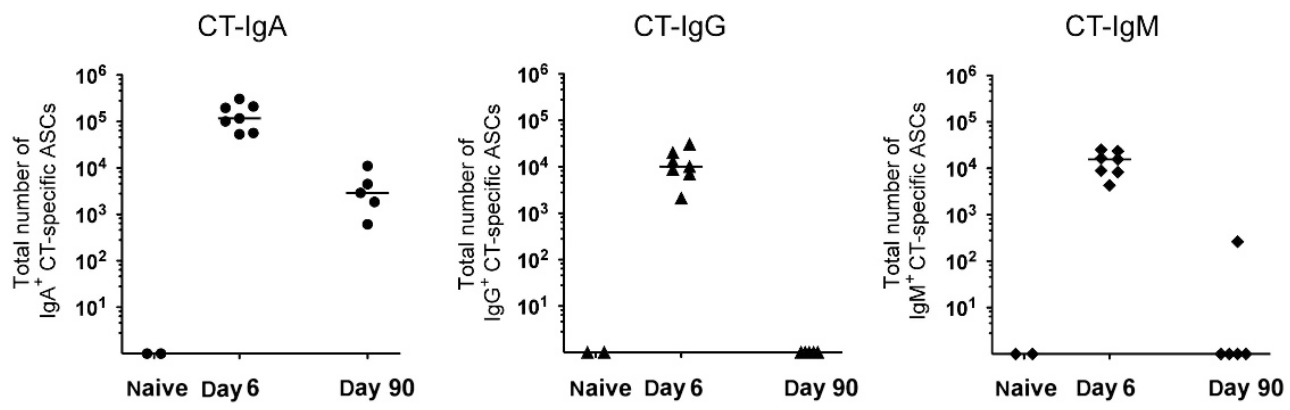

C
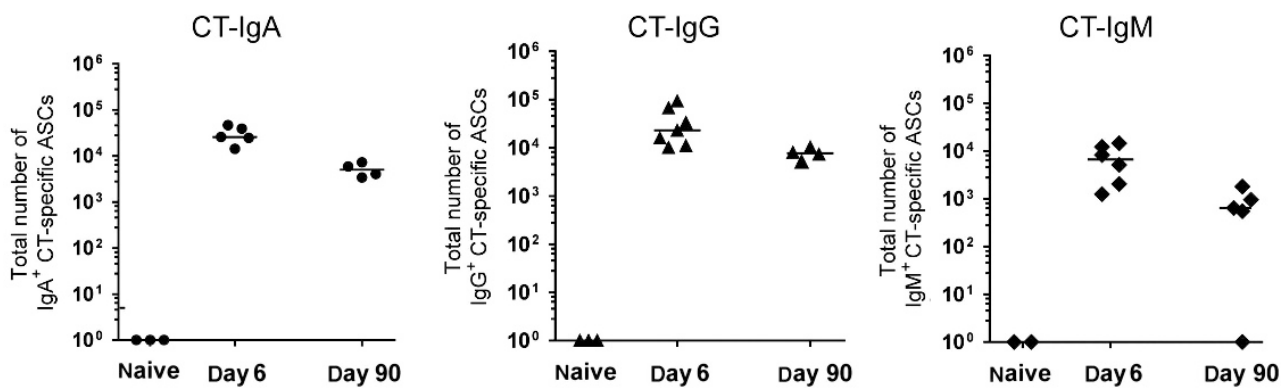

Figure 1 Toxin-specific plasma cells induced by oral immunization with cholera toxin (CT) persist in the lamina propria and the bone marrow. (a) C57BL/6 mice were immunized twice in a time interval of 21 days via oral gavage using $10 \mu \mathrm{g}$ cholera toxin. Lamina propria and bone marrow lymphocytes were isolated at the indicated time points and analyzed by ELISPOT for the presence of toxin-specific plasma cells. (b,c) Cholera toxin-specific plasma cells of the lamina propria (b) and the bone marrow (c) were determined by ELISPOT 6 and 90 days after oral boost immunization. Absolute numbers of cholera toxin-specific antibody-secreting cells (ASCs) are shown separately for immunoglobulin A (IgA), IgG, or IgM isotypes compared with non-immunized (naive) mice. Data points represent individual mice; data are pooled from three independent experiments. Bars indicate the median.

Next we analyzed PCs from the LP at later time points in order to determine their lifetime (Figure 4a). Using EdU labeling enabled us to detect long-lived PCs until the latest time point analyzed, namely, 9 months after boost by flow cytometry (Figure $4 \mathbf{b}, \mathbf{c}$ ), suggesting that PCs generated in mucosal immune responses have the potential to become long-lived.

\section{Gut epithelial cells are a major source of the PC survival factor APRIL}

By immunofluorescence histology, we confirmed the presence of $\mathrm{EdU}^{+} \mathrm{kappa}^{+}$cells in the small intestine 4 months (Figure 5a) and even up to 9 months after oral immunization, although at low abundance (data not shown). In line with the results obtained by flow cytometry, the majority of PCs at 4 months after boost were EdU-negative. However, we were able to detect scattered $\mathrm{EdU}^{+} \mathrm{IgA}^{+}$PCs in the diffuse LP.
Those long-lived intestinal PCs were localized in the villi as well as in the areas around the crypts (Figure 5a).

In the bone marrow, eosinophils have been shown to localize within the PC survival niche, where they are a source of APRIL. ${ }^{4}$ The detection of long-lived PCs in the LP prompted us to investigate whether a similar survival niche exists in the small intestine. The gut has a substantial population of eosinophils. ${ }^{23}$ In line with this, we could detect cells, which stained positive for the eosinophil marker major basic protein (MBP) in the diffuse LP (Figure 5b). A fraction of IgA ${ }^{+}$PCs was found to localize in contact with those $\mathrm{MBP}^{+}$cells in the gut by histology. However, the majority of PCs $(\sim 70 \%)$ were not found to contact eosinophils. Interestingly, this percentage did not differ between $\mathrm{EdU}^{+}$and EdU ${ }^{-}$PCs in EdU pulse-chase experiments 4 months after immunization (Figure $\mathbf{5 c}$,d). These results suggested that-unlike in the bone marrow-colocalization with eosinophils was not required for the survival of PCs in the 
a
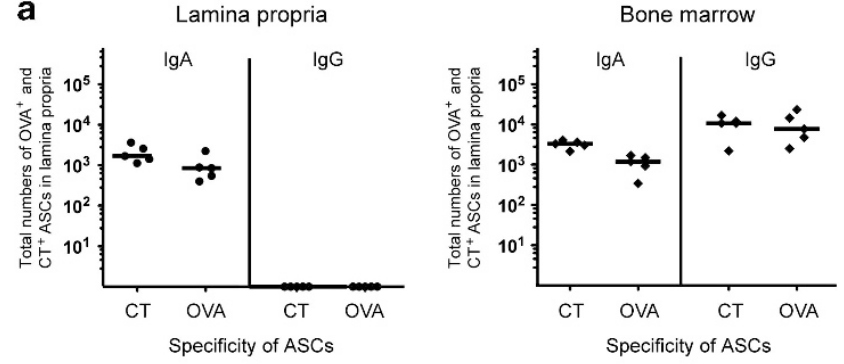

b

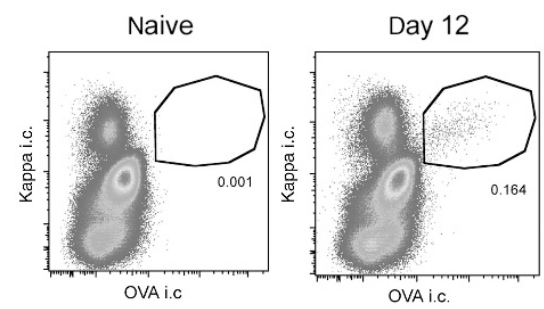

C

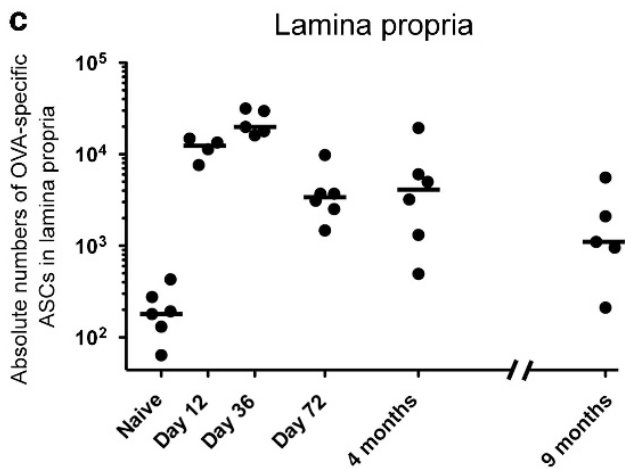

Time after secondary oral immunization

Figure 2 Mucosal-induced plasma cells specific against cholera toxin (CT) as well as the co-administered immunogen ovalbumin (OVA) persist in the lamina propria and the bone marrow at least for 9 months. (a) C57BL/6 mice were immunized twice in a time interval of 21 days via oral gavage using $10 \mu \mathrm{g} \mathrm{CT}$ and $100 \mathrm{mg}$ OVA. Nine months after boost, lamina propria and bone marrow lymphocytes were analyzed by ELISPOT for the presence of toxin-specific as well as OVA-specific plasma cells. Absolute numbers of CT- and OVA-specific antibody-secreting cells (ASCs) are shown per organ and depicted separately for immunoglobulin A $(\operatorname{IgA})$ and IgG isotypes, respectively. Data points represent individual mice from one experiment. Bars indicate the median. (b) C57BL/6 mice were immunized as described in a and lymphocytes from the lamina propria were isolated 12 days after oral boost to identify OVA-specific ASCs by flow cytometry. Cell suspensions were stained intracellularly with fluorochrome-coupled antibody against kappa light chain and fluorescently labeled OVA. Representative dot plots of immunized and naive mice indicate the identification of OVA-specific ASCs. Data are representative for 21 individual mice from five independent experiments. (c) C57BL/6 mice were immunized as described in a and intestinal lymphocytes were analyzed for the presence of OVA-specific ASCs as described in $\mathbf{b}$ at the indicated time points after oral boost. Absolute numbers of OVA-specific ASCs were calculated per mouse; data points represent individual mice; data are pooled from one to four independent experiments per time point. Bars indicate the median. The differences in absolute numbers of OVA-specific plasma cells between non-immunized controls and the various time points after boost were tested for statistical significance by Mann-Whitney $U$ test. All tests yielded significant differences: day 12 after boost $P=0.006$; day $36 P=0.003$; day $72 P=0.0095$; 4 months $P=0.001$; 9 months $P=0.01$.
LP. As shown by histology, eosinophils in the bone marrow are a major source of APRIL. ${ }^{4}$ We stained for this cytokine in the LP in order to evaluate its distribution in this tissue. The strongest signal for APRIL was detected in the gut epithelial cells (Figure 5e), in line with a recent publication. ${ }^{24} \mathrm{MBP}^{+}$ eosinophils as well as some other, yet unidentified cells in the LP were $\mathrm{APRIL}^{+}$, albeit the signal seemed less bright than in the epithelium. Taken together, our data indicate that in the gut-unlike in the bone marrow-colocalization with eosinophils within a survival niche has a minor role in the survival of long-lived PCs. Of note, eosinophils were reported to have a role for PC generation in the LP, and PC numbers in the gut have been shown to be reduced by about $50 \%$ in eosinophildeficient mice compared with wild-type mice, ${ }^{24}$ indicative of other sources of APRIL contributing to the presence of PCs at this location. Our findings indicate that the gut epithelium acts as the main provider of APRIL in the gut.

\section{PCs induced after oral immunization contribute to the long- lived PC pool in the bone marrow}

Plasma blasts have been shown to migrate from secondary lymphoid organs to the bone marrow in mice ${ }^{1,2}$ and humans ${ }^{25}$ during systemic immune responses. The bone marrow is the physiological site for the accumulation of long-lived PCs. It is thought to contain micro-environmental niches defined by a combination of stromal as well as hematopoietic cells, which supply the PCs with survival factors, thereby promoting their longevity. ${ }^{6}$ However, previous studies on long-lived PCs in the bone marrow were done using systemic challenges, and the mucosal and systemic PC pools have been considered to be compartmentalized. As we had found CT-specific ASCs in the bone marrow by ELISPOT in our previous experiments (Figure 1c), we next sought to confirm the presence of OVAspecific ASCs by flow cytometry up to 9 months after boost in the bone marrow (Figure 6a). To investigate whether mucosal immune reactions can elicit bona fide long-lived PCs in the bone marrow, we also analyzed EdU incorporation in bone marrow PCs in pulse-chase experiments. EdU ${ }^{+} \mathrm{kappa}^{+}$PCs were detected up to 9 months after oral boost in the bone marrow (Figure $\mathbf{6 b}, \mathbf{c}$ ). We confirmed the presence of $\mathrm{EdU}^{+}$ PCs in the bone marrow at 9 months after immunization by histology (Figure 6d). Notably, EdU was detected in OVAspecific PCs at this time point (Figure 6e).

The majority of antibodies secreted by PCs in the LP are shuttled into the gut lumen. ${ }^{26}$ In contrast, bone marrow PCs are responsible for the production of serum IgG and a fraction of serum IgA antibodies in mice. We could detect OVA-specific IgA as well as IgG in the serum of orally immunized mice up to 9 months after oral immunization, supporting the hypothesis that long-lived bone marrow PCs induced in mucosal immune responses contribute to the maintenance of long-term serum antibody titers (Figure 6f). We also examined the distribution of Ig isotypes within the $\mathrm{EdU}^{+}$and OVA-specific PC compartment by flow cytometry. Whereas, as expected, IgA was the dominant isotype of OVA-specific PCs in the LP, (median: 93.2\%: data not shown), there were almost equal fractions of 
a

OVA+CT OVA+CT

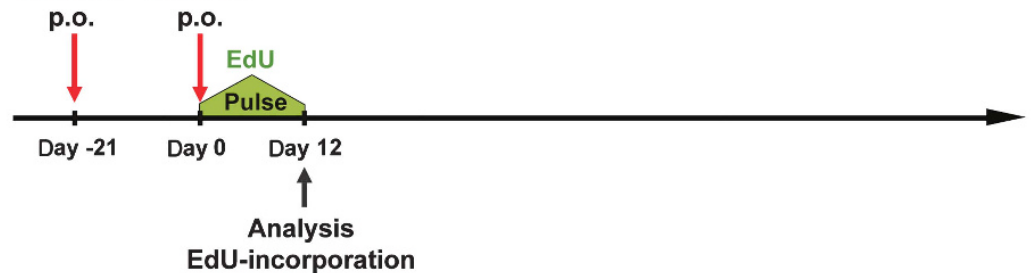

b Lamina propria

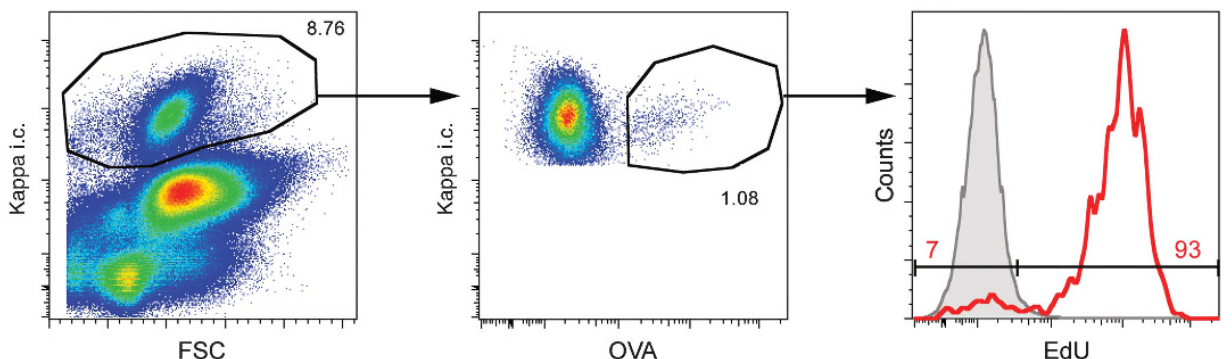

C Lamina propria
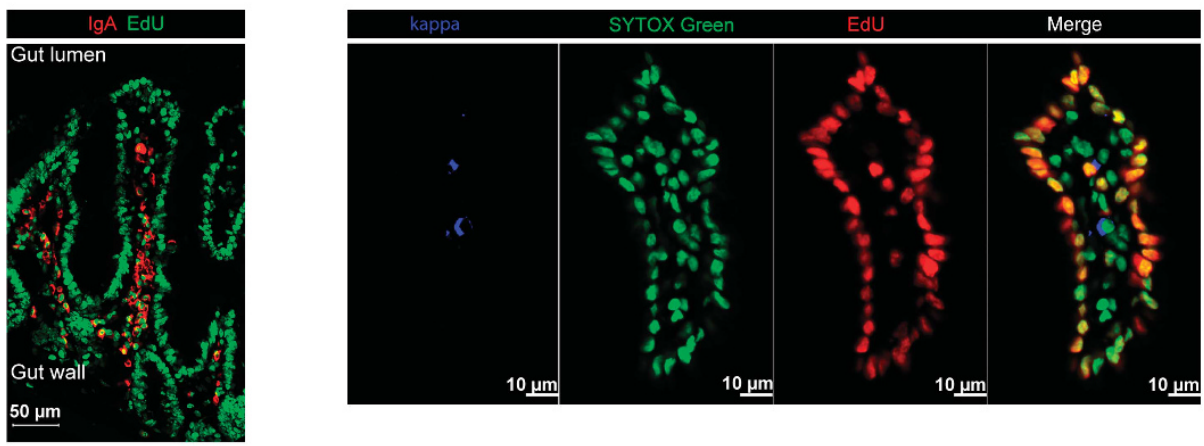

d Lamina propria
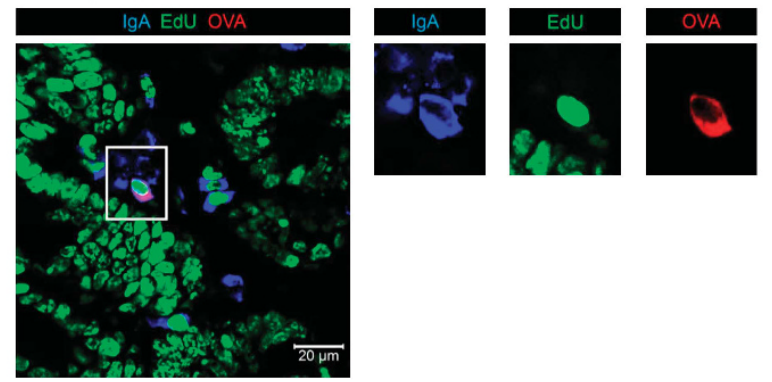

Figure 3 EdU (5-ethynyl-2'-deoxyuridine) incorporation by plasma blasts in the lamina propria. (a) C57BL/6 mice were immunized twice in a time interval of 21 days via oral gavage using $10 \mu \mathrm{g}$ cholera toxin (CT) and $100 \mathrm{mg}$ ovalbumin (OVA). To mark proliferating cells, mice received EdU continually in drinking water from day 0 (time point of oral boost) until day 12. (b) To investigate the incorporation efficiency of EdU, intestinal lymphocytes of the lamina propria were isolated at day 12, upon termination of EdU administration. Cells were stained intracellularly with fluorochrome-coupled antibody against kappa light chain and fluorescently labeled OVA. Staining of intranuclear incorporated EdU was performed using the Click-it EdU Alexa 647 Kit, and cells were analyzed by flow cytometry. After exclusion of dead cells via fixable eFluor 780 (not shown), representative dot plots indicate the OVAspecific plasma cell population. A representative histogram shows the EdU incorporation of OVA-specific plasma cells (red line) using intestinal plasma cells of non-EdU-fed mice (gray line) as negative control. (c) Intranuclear localization of EdU was confirmed 12 days after oral boost, directly after EdU administration via immunofluorescence microscopy. Cryosections of the small intestine were stained with anti-mouse IgA antibody, anti-mouse kappa light chain antibody, and fluorescently labeled anti-EdU antibody as well as SYTOX Green for visualization of nuclei. (d) OVA-specific, EdU ${ }^{+}$plasma cells in the lamina propria 12 days after oral boost. Cryosections of the small intestine were stained as described in c. FSC, forward scatter; IgA, immunoglobulin A.

OVA-specific $\operatorname{IgA}^{+}$and $\operatorname{IgG1}{ }^{+}$PCs present in the bone marrow at both day 12 and day 35 (see Supplementary Figure S2). This indicates that $\mathrm{IgG1}^{+} \mathrm{OVA}$-specific plasma blasts from mucosal immune responses preferentially home to the bone marrow compared with their $\operatorname{IgA}^{+}$counterparts and suggests that various isotypes generated in responses to different antigens vary in their capacity to migrate to the bone marrow. 


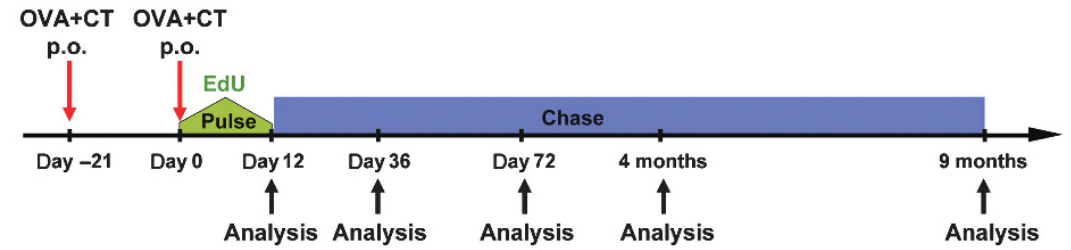

b Lamina propria
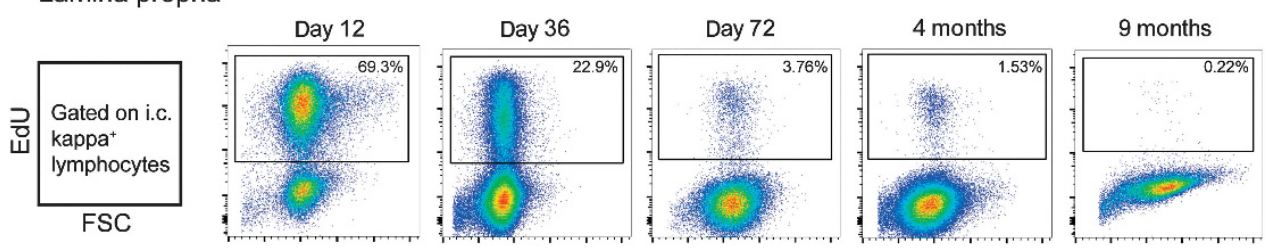

$\mathbf{C}$

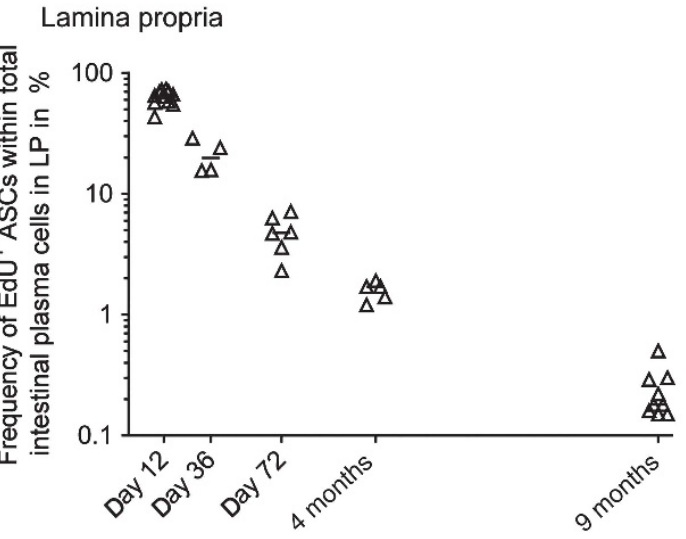

Figure 4 Identification of long-lived intestinal plasma cells by intranuclear incorporated 5-ethynyl-2'-deoxyuridine (EdU). (a) C57BL/6 mice were immunized and treated with EdU as described in (Figure 3a). After termination of EdU labeling, lamina propria (LP) lymphocytes were investigated by flow cytometry in the following EdU-free period (chase) at indicated time points for the detection of EdU ${ }^{+}$plasma cells, which represent long-lived plasma cells. (b) For identification and quantification of EdU-incorporating LP plasma cells via flow cytometry, isolated intestinal lymphocytes were stained intracellularly with fluorescently labeled antibodies binding the kappa light chain as well as intranuclear-incorporated EdU as described above in (Figure 3b). Representative dot plots permit differentiation of $\mathrm{EdU}^{+}$and EdU ${ }^{-}$subpopulations of intestinal plasma cells at indicated time points after oral immunization. Gating was defined for each time point using control mice that had not been fed with EdU. (c) Frequencies of EdU ${ }^{+}$fraction within total intestinal plasma cells were determined as decribed in $\mathbf{b}$ by flow cytometry until 9 months after oral immunization. Data points represent individual mice; data are pooled from one to three independent experiments per time point. Bars represent the median. ASC, antibody-secreting cell; CT, cholera toxin; FSC, forward scatter; OVA, ovalbumin.

\section{PCs generated after mucosal challenge accumulate and persist in the bone marrow without the participation of splenic B cells}

We detected a small fraction of OVA-specific IgA ${ }^{+}$PCs in the spleen at day 12 after immunization (data not shown). PCs specific for mucosal antigens have been reported to localize in the spleen, ${ }^{13}$ and the splenic marginal zone has recently been proposed as a crucial site for the induction of B-cell responses against gut-derived microbial antigens. ${ }^{27}$ We therefore tested whether the spleen is required for the presence of PCs in the bone marrow by comparing OVA-specific PC responses after oral immunization in splenectomized and control mice. As in the previous experiments, mice were fed with EdU for 12 days after an oral challenge with OVA and CT. PCs in single-cell suspensions from the LP and the bone marrow were identified by intracellular staining with fluorescently labeled OVA and antibodies against the kappa light chain and analyzed by flow cytometry at days 12 (LP and bone marrow) and 35 (bone marrow) after boost. There was a slight but significant increase in total PC numbers in both the LP (two times; Figure 7a) and bone marrow (three times; Figure $\mathbf{7 b}, \mathbf{c}$ ) of splenectomized animals at all time points investigated. This is probably an effect of the surgical manipulation in combination with the lack of the splenic niche for the PCs. However, the frequencies of OVAspecific PCs in the LP were similar in both the groups at day 12 (Figure 7d). This was reflected in similar frequencies of OVAspecific PCs in the bone marrow in both the groups at this time point (Figure 7e), indicating that migratory plasma blasts generated in a mucosal immune response are able to reach the bone marrow without participation of the spleen. At day 35, antigen-specific PC frequencies continued to be equal in both the groups (Figure 7f), indicating that PCs derived from mucosal immune responses are able to persist in the bone marrow without the involvement of the spleen. With respect to the $\mathrm{EdU}^{+} \mathrm{PCs}$, a slight but significant decrease in their frequency was detected at both the time points in the bone 
a
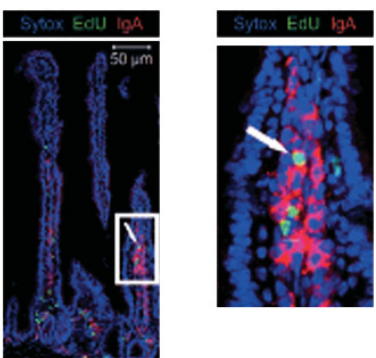

b
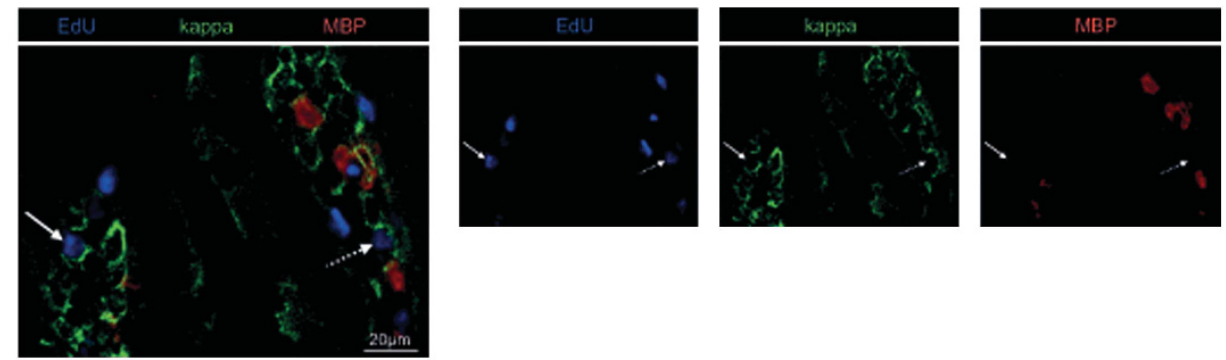

C

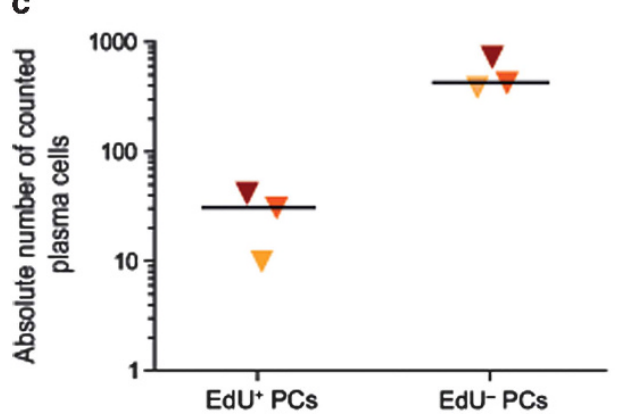

e

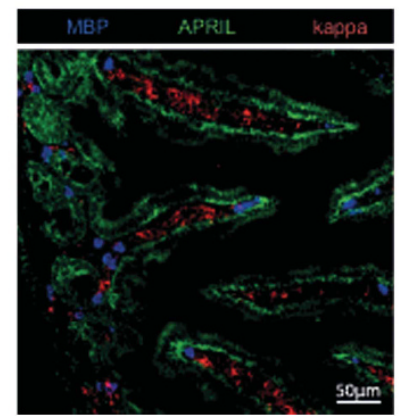

d
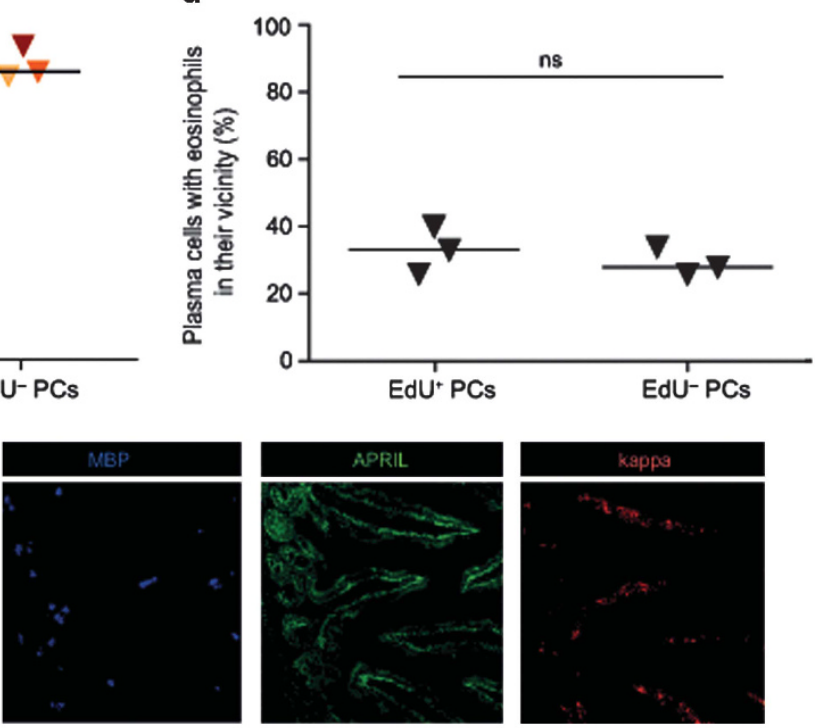

Figure 5 Localization and microenvironment of long-lived plasma cells (PCs) in the lamina propria. (a) Detection of long-lived PCs in the small intestine lamina propria by 5-ethynyl-2'-deoxyuridine (EdU) staining. Mice were immunized orally, fed with EdU for 12 days after boost, and analyzed 4 months later by confocal microscopy. The left confocal micrograph shows villi stained with a nuclear dye (blue) and fluorescently labeled antibodies binding immunoglobulin A (IgA; red) and EdU (green) to identify long-lived PCs. The right panel represents a zoom of the white boxed area; the arrow denotes an $\mathrm{EdU}^{+}$long-lived PC. (b) Immunofluorescence microscopy of lamina propria 4 months after oral boost. Cryosections of the small intestine were stained with antibodies against EdU (blue), kappa light chain (green) to identify PCs, and major basic protein (MBP, red) as a marker for eosinophils. Colocalization of eosinophils and PCs was defined as $<5-\mu \mathrm{m}$ distance between them. Solid arrow indicates an EdU ${ }^{+} \mathrm{PC}$ not in contact with eosinophils; dotted arrow marks a PC in the vicinity of an eosinophil. (c) PCs from histological sections of the small intestine 4 months after boost immunization were counted and evaluated for their EdU incorporation. Numbers of $\mathrm{EdU}^{+}$and EdU ${ }^{-} \mathrm{PCs}$ are shown, enumerated from four to eight sections from three individual mice. Each color represents one animal. Total PC numbers counted per animal were 779 (red), 397 (yellow), and 456 (orange). Bars represent the median. (d) Frequency of PCs with eosinophils in their vicinity. The number of $\mathrm{EdU}^{+}$and $\mathrm{EdU}^{-}$antibody-secreting cells was counted and their vicinity to eosinophils (within a radius of $5 \mu \mathrm{m}$ around the outer border of the PCs) was determined. The plot shows the percentage of PCs in contact with eosinophils in the lamina propria among $\mathrm{EdU}^{+}$and $\mathrm{EdU}^{-} \mathrm{PCs}$. Bars represent the median. No significant difference with respect to eosinophil colocalization was detected among the $\mathrm{EdU}^{+}$and $\mathrm{EdU}^{-}$population using the two-tailed Mann-Whitney U-test. (e) Immunofluorescence microscopy of the lamina propria at 4 months after oral boost reveals a strong APRIL (A proliferation-inducing ligand) signal in the epithelium. Cryosections of the small intestine were stained with antibodies against APRIL (green), kappa light chain (red) to identify PCs, and MBP (blue) as a marker for eosinophils. NS, not significant. 
marrow (Figure $7 \mathbf{h}, \mathbf{i}$ ), whereas their frequency was equal in the LP of both cohorts (Figure $7 \mathbf{g}$ ). Notably, there was no difference in the frequency of the $\mathrm{EdU}^{+}$fraction among the OVA-specific PCs in both the organs at day 12 and in the bone marrow at day 35 (Figure $7 \mathbf{j}-\mathbf{1}$ ). Splenic B cells did not have a crucial role in the isotype composition of the bone marrow compartment as the frequencies for all three isotypes tested did not differ significantly between splenectomized and control cohorts at both the time points (see Supplementary Figure S3A,B).

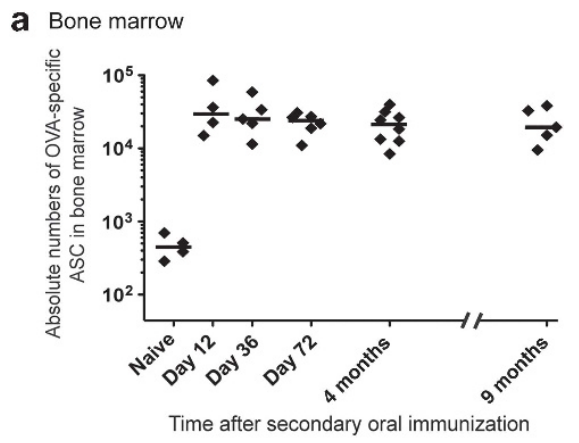

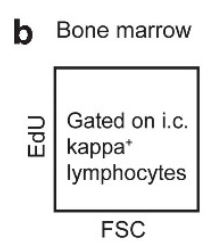

C

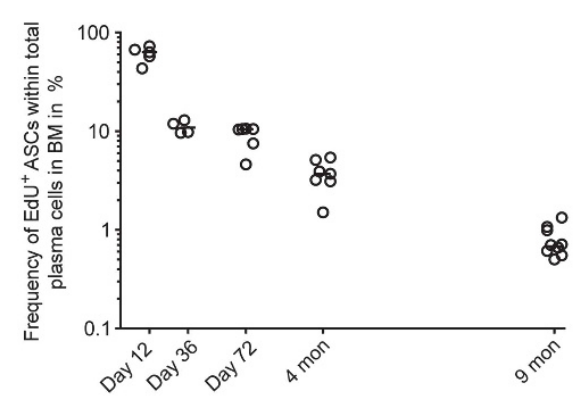

e
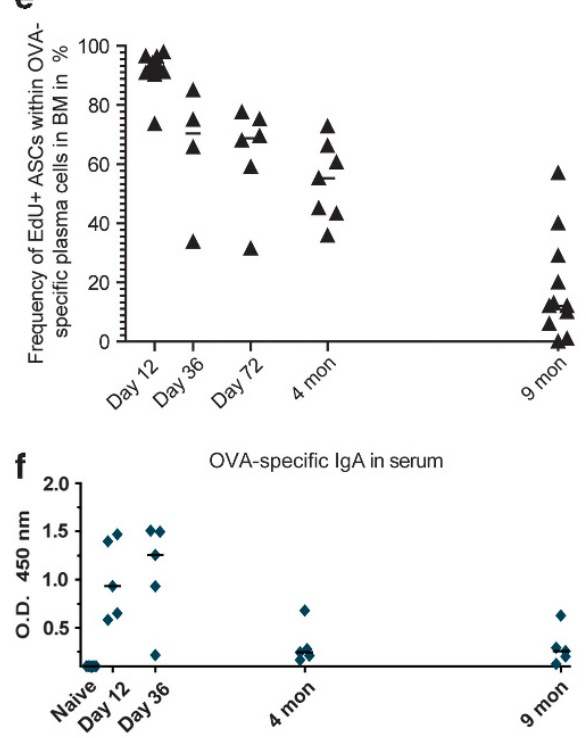

d

Bone marrow at the time point 9 months
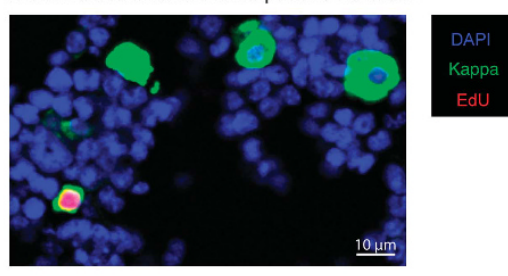

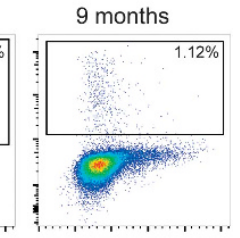

i

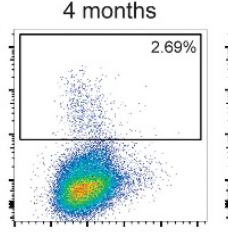

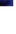




\section{Bone marrow PCs secrete antibodies specific for components of commensal bacteria}

Having found that the bone marrow compartment includes PCs specific for orally administered antigens, we next investigated whether there are also antibodies against mucosal antigens, such as components from commensal bacteria produced in this compartment. To this end, we tested the reactivity of bone marrow PCs from individual mice against bacterial lysates isolated from stool samples of littermates by ELISPOT. In humans, an increased permeability of the gastrointestinal barrier has been associated with a higher level of systemic antibodies against bacteria in the gut. ${ }^{28,29}$ As CT is known to cause permeability of the intestinal barrier, we therefore examined the anti-stool lysate reactivity of PCs from mice that had received CT orally twice (56 and 35 days before analysis). PCs producing IgM as well as class-switched isotypes (IgA and IgG) were detected in comparable numbers among bone marrow PCs in both CT-treated mice and in untreated controls (see Supplementary Figure S4A,B). From these experiments, we conclude that the bone marrow compartment includes PCs specific for commensal bacteria components and that the ability to mount these responses is independent from a barrier leak in the intestine. In this respect, it should be noted that we also detected $\mathrm{EdU}^{+} \mathrm{IgA}^{+}$PCs in the bone marrow in unimmunized mice kept under specific pathogen-free conditions (see Supplementary Figure S4C,D), which might include long-lived cells secreting antibodies directed against structures from commensal bacteria.

\section{DISCUSSION}

Long-lived PCs persist in the bone marrow, where they are located in survival niches that are defined by a combination of cellular and molecular factors. Together, these factors promote PC survival, enabling them to provide long-term antibody titers over an individual's lifespan. ${ }^{18,30}$ Whereas the existence of long-lived bone marrow PCs and their contribution to protective as well as pathogenic antibody titers has been shown extensively in systemic immune responses, ${ }^{31}$ the situation is less clear for PCs generated in mucosal immune responses.
Here we show that, following oral immunization with CT, PCs can be detected over at least 9 months in the diffuse LP. Moreover, if CT and OVA are co-administered, the adjuvant activity of CT promotes the generation of long-lived OVAspecific PCs in the LP, which are detected over even longer periods, at least 9 months after immunization. Although the majority of ASCs in the small intestine are considered to be short-lived, ${ }^{14}$ there is accumulating evidence in the literature that PCs specific for a certain antigen are present at late time points after antigen challenge in the LP. Specifically, this has been shown in mice for PCs not only specific for $\mathrm{CT}^{32}$ but also for commensal bacteria, ${ }^{17}$ as well as in human tissue cultures derived from the small intestine. ${ }^{16}$ In mucosal immune responses, Toll-like receptor stimulation has been suggested to trigger $\mathrm{B}$-cell activation independent of $\mathrm{B}$ cell receptor stimulation, $^{33}$ thus PCs present at late time points after challenge could be generated de novo from activated B cells, even independent of the presence of their antigen. However, our work for the first time demonstrates that PCs can persist in vivo in the LP without proliferation, as shown by EdU positivity 9 months after the cessation of EdU administration. We show that a fraction of PCs in the LP can persist despite a strong competition for survival factors coming from short-lived PCs, which are generated constantly in the intestine of live animals. Hence, LP PCs are able to become long-lived, similar to PCs in the bone marrow, and suggesting that survival niches similar to the ones in the bone marrow are present in the LP. Hapfelmeier et al. ${ }^{17}$ suggested that the turnover of PCs in the LP is regulated by attrition, indicating that the level of antigenic stimulation regulates the lifespan of these cells via the generation of new PCs. A similar concept has been discussed for bone marrow PCs: newly generated migratory plasma blasts have been suggested to compete with resident PCs for a limited number of available survival niches. ${ }^{6,34}$ To maintain resident PCs at sufficient numbers to provide protection and to accommodate as many new specificities as possible, an optimal influx rate of new PCs to the bone marrow per immune response was estimated to be three times higher than the minimally required number of PCs providing protection. ${ }^{35}$ It will be interesting to

Figure 6 Mucosal-induced plasma cells contribute to the long-lived plasma cell pool in the bone marrow (BM). (a) C57BL/6 mice were immunized twice in a time interval of 21 days as described above. Lymphocytes of the BM were isolated at the indicated time points, stained intracellularly with fluorochrome-coupled antibody against kappa light chain and fluorescently labeled ovalbumin (OVA), and were analyzed by flow cytometry. Absolute numbers of OVA-specific antibody-secreting cells (ASCs) of the BM were calculated per mouse; data points represent individual mice; data are pooled from one to three independent experiments per time point. Bars indicate the median. The differences in absolute numbers of OVA-specific plasma cells between non-immunized controls and the various time points after boost were tested for statistical significance by Mann-Whitney $U$-test. All tests yielded significant differences: day 12 after boost $P=0.03$; day $36 P=0.02$; day $72 P=0.01$; 4 months $P=0.004$; and 9 months $P=0.02$. (b) To analyze 5ethynyl-2'-deoxyuridine (EdU) incorporation of BM plasma cells, isolated lymphocytes were stained intracellularly for kappa light chain and intranuclear-

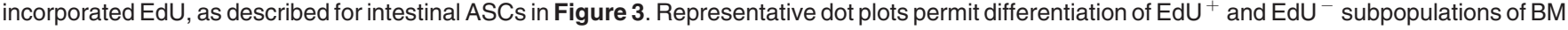
plasma cells at the indicated time points after oral immunization. Gating was defined for each time point using control mice that had not been fed with EdU. Results are representative of 4-9 individual mice from one to three independent experiments per time point. (c) Frequencies of EdU ${ }^{+}$ASCs within total BM plasma cells were determined as described in $\mathbf{b}$ by flow cytometry. Data points represent individual mice; data are pooled from one to three independent experiments per time point. Bars represent the median. (d) EdU ${ }^{+}$plasma cell in the BM 9 months after oral boost. Cryosections of the BM were stained for kappa light chain, EdU, and DAPI (4,6-diamidino-2-phenylindole) to visualize nuclei. (e) Frequencies of EdU ${ }^{+}$fraction within OVAspecific BM plasma cells. Data points represent individual mice; data are pooled from one to three independent experiments per time point. Bars represent the median. (f) Systemic OVA-specific antibodies of immunoglobulin A (IgA) and IgG isotypes, respectively, were analyzed by serum enzyme-linked immunosorbent assay at the indicated time points until 9 months after oral boost. Optical density (O.D.) was determined at $450 \mathrm{~nm}$. Data points represent individual mice from one experiment. Bars represent the median. FSC, forward scatter. 
- Control animals o Splenectomized animals

a
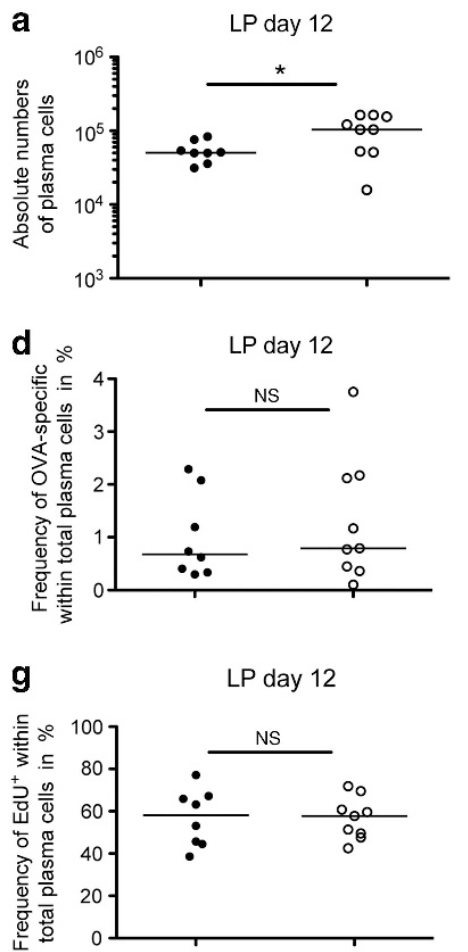

j

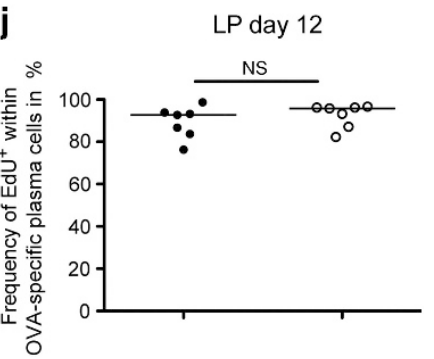

b

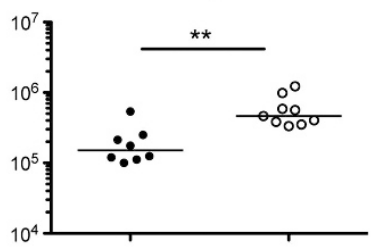

e

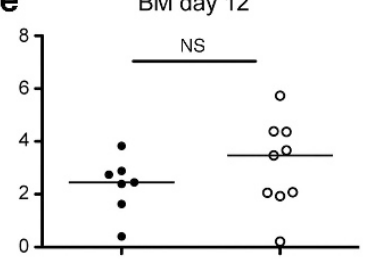

h BM day 12

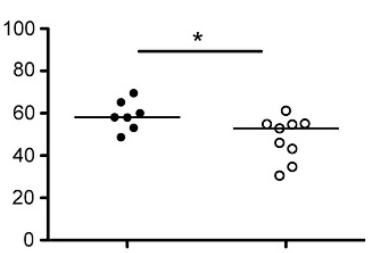

$\mathbf{k}$

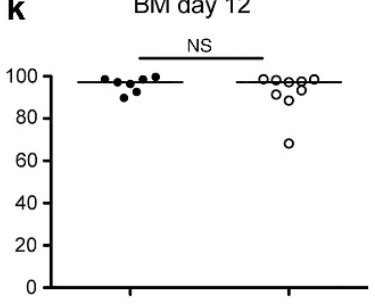

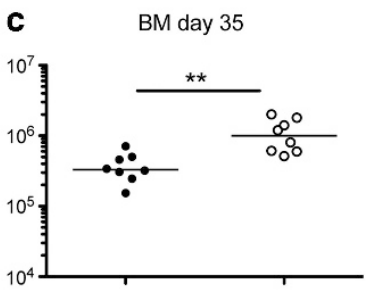
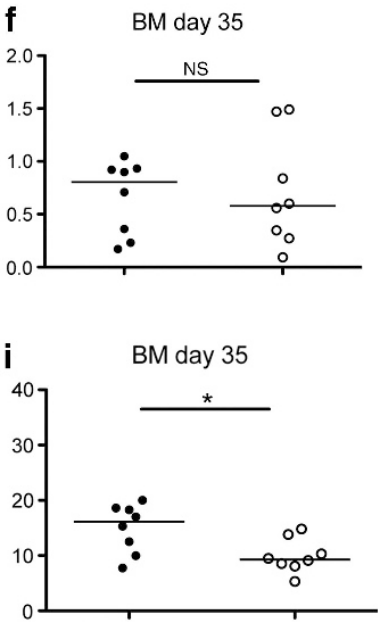

BM day 35

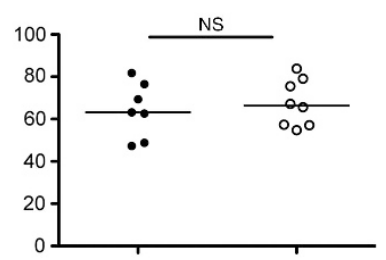

Figure 7 Analysis of the plasma cell response in splenectomized animals. (a-c) C57BL/6 mice were immunized twice in a time interval of 21 days as described before. In one cohort, a splenectomy was performed 2-3 weeks before the first immunization. Lymphocytes from the bone marrow (BM) and the lamina propria (LP) were stained intracellularly with fluorochrome-coupled antibody against kappa light chain and analyzed by flow cytometry. Absolute numbers of total antibody-secreting cells of the LP at the day 12 (a) and the BM at days 12 and 35 (b,c) after immunization were calculated per mouse. Data points represent individual mice pooled from three-four independent experiments per time point. The differences in absolute numbers of plasma cells between non-splenectomized controls and splenectomized animals were tested for statistical significance by Mann-Whitney U-test. All tests yielded significant differences $\left({ }^{\star} P<0.05 ;{ }^{* *} P<0.01\right)$. (d-f) Frequencies of the ovalbumin (OVA)-specific fraction within total LP plasma cells at day 12 (d) and BM at days 12 and 35 (e and f) in splenectomized and non-splenectomized mice were determined as described in Figure 6a. Data points represent individual mice; data are pooled from three to four independent experiments per time point. Bars represent the median. The differences in frequencies of OVA-specific plasma cells between non-splenectomized controls and splenectomized animals were analyzed for statistical significance by MannWhitney U-test. All tests yielded no significant differences. (g-i) Frequencies of EdU ${ }^{+}$(5-ethynyl-2'-deoxyuridine positive) fraction within total total plasma cells in LP at day $12(\mathbf{g})$ and BM at days 12 and 35 ( $(\mathbf{g}$ and $\mathbf{i})$ in splenectomized and non-splenectomized mice were determined as described in Figure 3 and Figure 6. Data points represent individual mice, data are pooled from three to four independent experiments per time point. Bars represent the median. The differences in frequencies of $\mathrm{EdU}^{+}$plasma cells between non-splenectomized controls and splenectomized animals were tested for statistical significance by Mann-Whitney $U$-test. Tests for BM yielded significant differences at both time points $(P<0.05)$. In LP, there was no significant difference. (j-I) Frequencies of the EdU ${ }^{+}$fraction within OVA-specific plasma cells in LP at day 12 (j) and BM at days 12 and 35 (k, I) in splenectomized and non-splenectomized mice. Data points represent individual mice; data are pooled from three to four independent experiments per time point. Bars represent the median. The differences in frequencies of EdU ${ }^{+}$plasma cells within OVA-specific plasma cells between non-splenectomized controls and splenectomized animals were tested for statistical significance by Mann-Whitney U-test. All tests yielded no significant differences. NS, not significant.

determine these parameters in the LP as in this case, unlike in the bone marrow, induction sites and persistence sites for PCs are localized within the same tissue. Therefore, a much stronger competition for niches is expected, and it will be important to determine whether certain PC specificities are more prone to survive under these conditions as others.
In the bone marrow, eosinophils have been described as a crucial cellular component of the PC survival niche. ${ }^{4}$ They produce APRIL, which is known to promote PC survival by binding to B-cell maturation protein. This results in the production of the anti-apoptotic protein myeloid cell leukemia $1,{ }^{36}$ which sustains PC survival. Genetic deficiency of eosinophils 
results in the depletion of about $70 \%$ of long-lived bone marrow PC in vivo, underlining the role of eosinophils in promoting bone marrow PC survival. ${ }^{4}$ In our experiments, one-third of the ASCs localize in the vicinity of eosinophils in the LP. This percentage did not differ between long-lived and short-lived PC populations (Figure 5c). However, APRIL acts as a soluble survival factor, and we have recently shown that in the bone marrow eosinophils are not required to make prolonged contact with PCs to promote their survival. ${ }^{37}$ In contrast to PCs and stromal cells, which are static components of the PC survival niche, eosinophils are dynamic elements of this niche, and are exchanged frequently, thereby explaining a low rate of direct contact counts. ${ }^{37}$

Moreover, the major sources of APRIL relevant for PC survival may differ between various tissues. Although APRIL ${ }^{+}$ eosinophils were detected in the LP by histology, the strongest signal intensity was detected in the epithelium, consistent with published data from humans ${ }^{38}$ and mice. ${ }^{24}$ Consistent with the concept that eosinophils are not the sole source of APRIL in the LP, a total absence of PCs in the LP of either eosinophildeficient or eosinophil-depleted mice was not observed but rather a $\sim 50 \%$ reduction in PC numbers, concurrent with a similar reduction in luminal and fecal IgA titers. Notably, in addition to the effect on PCs, Chu et al. ${ }^{24}$ also found that eosinophils were able to promote the generation of $\operatorname{IgA}^{+} \mathrm{B}$ cells in the Peyer's Patches. As PCs were obviously already affected at the point of their generation, a conclusion about the role of eosinophil-derived APRIL regarding their maintenance could not be drawn in vivo. The authors describe a crucial effect of eosinophils for the survival of LP-derived PCs as demonstrated by in vitro co-cultures, but the contribution of epithelial cells was not investigated. Taken together, these data show that eosinophils are not the only source of APRIL in the intestine. They suggest a role for the intestinal epithelium in contributing to the PC survival niche in the LP by producing APRIL and thus a different structure of the PC survival niche in various organs.

In addition to APRIL, other molecules have been shown to promote PC survival in the bone marrow. These include interactions via $\mathrm{CD} 28$ on the PCs with CD80 on dendritic cells in the bone marrow as well as interleukin- 6 and the chemokine CXCL12, which are derived from stromal cells. In the future, it will be important to investigate which types of cellular interactions have a role for sustaining PC survival in the gut and whether there are intrinsic molecular similarities and differences in the long-lived PC compartments of both organs.

Our experiments further show that long-lived PCs generated in an immune response against orally delivered antigens are not only restricted to the mucosal tissue but can also be found in the bone marrow, thereby contributing to the long-lived PC pool at this site. We show here that a fraction of long-lived PCs generated after a mucosal challenge persist over comparable time spans as PCs generated in systemic immune responses and that these cells produce antibodies but do not proliferate extensively.

Whether PCs generated in mucosal immune responses occupy bone marrow niches distinct from the ones originating in systemic responses was not investigated here. In our preliminary histological analysis, we could not detect obvious differences in the location between $\mathrm{IgA}^{+}$and $\mathrm{IgG}^{+}$bone marrow PCs. However, at least in humans, circulating $\operatorname{IgA}{ }^{+}$ plasma blasts are equipped with chemokine receptors and adhesion molecules that are typical for cells generated in mucosal immune responses (the gut-homing integrin $\alpha_{4} \beta_{7}$ as well as chemokine receptors CCR9 and CCR10) and therefore differ from their counterparts in systemic immune responses, which mainly express integrin $\alpha_{4} \beta_{2}$, CXCR3, and CXCR $4 .{ }^{34}$ It will be crucial to investigate whether they keep this mucosal adhesion molecule/chemokine receptor signature once they have reached their niche in the bone marrow, as this would suggest different requirements of mucosal and systemic PCs with respect to the signals that are available to the PCs in the niches.

It is believed that the mucosal and the systemic PC compartment in mice are compartmentalized; IgA production to orally administered antigens is thereby largely restricted to the intestine. However, as our data show, at least under certain conditions, the compartmentalization between mucosal and systemic compartments may be less strict than previously thought. In this context, it is notable that seminal work from the early 1970s has shown monomeric serum IgA to be derived from the bone marrow in humans (reviewed in Heremans ${ }^{39}$ ). Considerable differences between species exist in this context, as in mice the largest fraction of total serum IgA is thought to predominantly originate from the gut. However, even if the fraction of IgA produced in the gut outnumbers the bone marrow-derived serum IgA, our experiments clearly show that long-lived PCs of IgA as well as IgG isotypes specific for intestinal antigens can be deposited in the bone marrow and contribute to serum Ig levels and therefore form a part of humoral memory. The protective potential of these antibodies against gut-derived and systemic antigens, and thus their impact on vaccinology, will be the subject of further studies. The migration of plasma blasts originating from mucosal immune responses to the bone marrow has been reported previously in murine immune responses against bacteria ${ }^{40}$ and viruses. ${ }^{13}$ Importantly, to our knowledge the data presented here demonstrate for the first time that splenic B-cell activation (which has been implied in systemic B-cell responses against commensal bacteria ${ }^{27}$ ), is not necessary for the generation of long-lived bone marrow PCs specific for orally administered antigens. These long lived PCs in the bone marrow contribute to systemic humoral memory, as reflected in the presence and persistence of OVA-specific antibody titers in the serum (Figure 6f) and OVA-specific PCs in the bone marrow (Figure 2a and Figure 6a). We also found PCs specific for components of commensal bacteria in the bone marrow. As a significant fraction of these cells were actually class-switched, including IgG (see Supplementary Figure S4A,B), we can exclude that these antibodies have a preimmune origin. The presence of class-switched serum antibodies against commensals has been described in humans, and these antibody levels remained stable over several years. ${ }^{28,29}$ In contrast, classswitched serum antibodies against colonizing bacteria in the 
intestine were not detected in mice. ${ }^{41,42}$ Notably, the levels of serum antibodies against various bacteria differed significantly between human individuals, and increased levels were detected in inflammatory bowel disease patients compared with healthy individuals, suggesting a role for the gastrointestinal barrier function in determining the titer of anticommensal antibodies. ${ }^{29}$ This suggested the systemic dissemination of commensal microbes due to a disturbed barrier as cause of the systemic response. A similar effect was postulated in a mouse model of intestinal barrier dysfunction. ${ }^{43}$ In contrast, we could not find isotype differences in CT-immunized vs. untreated cohorts (see Supplementary Figure S4B); however, we did not perform a detailed comparison of the microbiome in mice of both cohorts. The potential of a bacterial strain to elicit a systemic response may not only depend on the ability of the bacterium to translocate via the gastrointestinal barrier but also on the signal strength that a B-cell receives upon activation. Various commensal strains may thus differ in their capacity to activate $\mathrm{B}$ cells in the mucosa, depending on whether they are strict commensals or have capacities of pathobionts. ${ }^{44}$ Although the mice used in our studies were kept under specific pathogen-free conditions, the commensal microbiota of the mice in our facility are likely to differ from the microbiota of mice used in the previous studies, which could account for the observed differences, as recent studies have shown that the commensal microbiota in different specific pathogen-free facilities can critically influence systemic immune reactions. ${ }^{45}$ Future studies will be required to determine the specificity of these antibodies, as currently we cannot exclude that they are directed against conserved bacterial structures. Importantly, we had to culture the bacteria derived from the gut in order to have a sufficient amount for the preparation of lysates for ELISPOT detection. This very likely caused a reduction in bacterial diversity, which may have actually led to an underestimation of the number of PCs specific for bacterial components in our experiments, a question which also needs to be addressed in further experiments.

We observed a decrease in the $\mathrm{EdU}^{+}$fraction among OVA-specific PCs in the bone marrow over time (Figure 6e). This might reflect an ongoing proliferation in OVA-specific B cells that could, on the one hand, be caused by persistence of antigen, on the other hand, their activation could be triggered by Toll-like receptors as has been suggested before, ${ }^{33,46}$ in particular in memory B cells. ${ }^{47}$ In this context, it should be noted that we have actually observed $\mathrm{EdU}^{+} \mathrm{B}_{2} 20^{+}$cells localized within Peyer's Patches up to at least 72 days after immunization (data not shown), which could represent memory $\mathrm{B}$ cells in this tissue. Further investigations to characterize this population are underway. Notably, a recent publication by Pabst and co-workers demonstrated that, after Bortezomibinduced PC depletion, the repertoire of the repopulating LP PCs remained remarkably stable in individual mice, indicating a recall of already existing specificities among these individuals. ${ }^{48}$ This may reflect the activation of memory B cells in the LP, which are not sensitive to Bortezomib-induced depletion. ${ }^{31}$ In this report, we did not investigate which signals actually trigger the proliferation of OVA-specific B cells at late time points after immunization. However, our point is that longlived PCs are in principle able to persist in the LP and that mucosally induced PCs contribute to the long-lived compartment in the bone marrow. The possibility of residual antigen leading to the de novo generation of OVA-specific PCs by residual antigen does not change this interpretation, as longlived PCs are unequivocally identified in our experiments by EdU pulse labeling, but it might lead to an underestimation of the fraction of long-lived cells among the OVA-specific PCs in our experiments.

Our findings also underline the potential of $\mathrm{CT}$ as a mucosal adjuvant, ${ }^{20}$ as not only CT-specific but also OVA-specific ASCs persisted in both LP as well as bone marrow when CT was co-administered as adjuvant. The potential of CT to elicit longlived systemic PC responses may have an impact for the development of vaccines. A fusion protein comprising the CTA1 moiety of CT and a dimer of the $S$. aureus protein $\mathrm{D}$-fragment has been shown to promote long-term humoral memory development in mice compared with other adjuvants by promoting germinal center responses, with an effect on both memory B-cell and PC numbers. ${ }^{49}$ Certain differences exist between the murine and human mucosal immune systems, which, with respect to humoral immunity, include IgA subclass differences (one single IgA subclass in mice vs. two in humans ${ }^{50,51}$ ), the presence of a specific Fc receptor solely for IgA (Fc $\alpha \mathrm{R} 1)$ in humans, ${ }^{52}$ as well as the dominance of polymeric IgA in the body fluids and transport of polymeric IgA from the circulation into the gut via the hepatobiliary route in mice, whereas in humans IgA is not transported from the circulation into external secretions. ${ }^{53}$ These differences have to be taken into account with respect to the effect of bone marrow-derived antibodies on the intestinal immune system. However, the finding that plasma blasts generated in mucosal immune responses can have an extended lifetime under certain conditions and can contribute to systemic Ig production by joining the long-lived bone marrow PC pool has to be considered for protective as well as for pathogenic humoral immune responses.

\section{METHODS}

Mice and immunizations. C57BL/6 mice were purchased from Charles River (Sulzfeld, Germany) and kept under specific pathogenfree conditions in the animal facility of the DRFZ, Berlin. For some experiments, mice were bred in the DRFZ facility at the Bundesinstitut für Risikoforschung in Berlin. Adult mice (aged between 7 and 13 weeks) were immunized with $100 \mathrm{mg}$ OVA (Sigma-Aldrich, St Louis, MO) and $10 \mu \mathrm{g}$ CT (List Biological, Campbell, CA) in $200 \mu \mathrm{l}$ phosphate-buffered saline (PBS) using a feeding needle that was placed through the mouth into the stomach. Prior to the immunization, gastric acid was neutralized by applying a $50 \%$ saturated sodium bicarbonate solution via the feeding needle. Mice were fed with EdU (Life Technologies, Carlsbad, CA) via drinking water $\left(0.5 \mathrm{mg} \mathrm{ml}^{-1}\right)$ constantly over a time period of 12 days after boost immunization. To ensure individual intake and fresh supply, EdU was changed every 3 days. All animal experiments were conducted according to German animal protection laws and approved by the appropriate governmental authority (Landesamt for Gesundheit und Soziales) in Berlin.

Splenectomy. Mice were anesthetized by intraperitoneal injection of Ketamine and Xylazine. The abdominal cavity was opened by a 
subcostal incision on the left flank. The spleen was located and the splenic vessels were ligated and cauterized. After removal of the spleen, the abdominal wall and skin were sutured with absorbable sutures (Surgicryl Rapid 6.0, SMI AG, Crombach, Belgium). A single dose of Enrofloxacin was administered prophylactically subcutaneously.

Cell isolation. To harvest cells from the bone marrow, femora and tibiae were cleaned from muscle tissue, epiphyses were removed, and the bone marrow was flushed out with RPMI using a syringe with a 27$\mathrm{G}$ needle attached. The resulting suspensions were mechanically disrupted in RPMI with a syringe plug and filtered through a $70-\mu \mathrm{m}$ cell strainer. Erythrocytes were lysed by adding $\mathrm{NH}_{4} \mathrm{Cl}$ at room temperature, and then cells were washed in cold $\left(4{ }^{\circ} \mathrm{C}\right) \mathrm{PBS} / 0.5 \%$ bovine serum albumin (BSA). Prior to the isolation of lymphocytes from the small intestine LP, Peyer's patches were removed under a stereomicroscope. The small intestine was opened longitudinally, cut into small $(5 \mathrm{~cm})$ pieces, and washed in RPMI for $30 \mathrm{~min}$ at $37^{\circ} \mathrm{C}$ under constant stirring to remove the mucus. Subsequently, intraepithelial lymphocytes were removed by vigorous shaking. After washing with RPMI, the tissue was digested twice for $15 \mathrm{~min}$ at $37^{\circ} \mathrm{C}$ in $40 \mathrm{ml}$ RPMI containing collagenase $\mathrm{D}\left(0.12 \mathrm{U} \mathrm{ml}^{-1}\right)$ and collagenase VIII $\left(206 \mathrm{U} \mathrm{ml}^{-1}\right)$. The digestion was stopped by adding cold $\left(4^{\circ} \mathrm{C}\right)$ RPMI and EDTA, and then cells were passed through a $70-\mu \mathrm{m}$ cell strainer. Lymphocytes from Peyer's patches and mesenteric lymph nodes were disrupted in PBS/0.5\% BSA with a syringe plug, and the cell suspension was then filtered through a $70-\mu \mathrm{m}$ cell strainer.

Flow cytometry. Single-cell suspensions were blocked with antibodies against Fc-receptors (CD16/32, clone 2.4G2, DRFZ) and stained with the following antibodies: rat anti-mouse CD45.2-APC (clone 104, eBiocience, San Diego, CA), rat anti-mouse CD138-PE (clone 281-2, BD Pharmingen, Franklin Lakes, NJ), rat anti-mouse B220-PE-Cy7 (clone RA3-6B2, BioLegend, San Diego, CA), rat anti-mouse kappa light chain (clone 187.1) coupled to Pacific Blue, FITC, Alexa 594 or Alexa 647 (DRFZ), polyclonal rat anti-mouse IgA-Pacific Orange (Southern Biotech, Birmingham, AL, conjugated at the DRFZ), rat anti-mouse IgA-PE (clone 11-44-2, Southern Biotech), rat anti-mouse IgM-PE-Cy7 (clone II/41, eBioscience), and rat anti-mouse IgG1BV421 (A85-1, BD Pharmingen). Prior to intracellular staining of immunoglobulins, samples were fixed and permeabilized (Cytofix/ Cytoperm Kit, BD). Dead cell exclusion on fixed samples was performed using the fixable viability dye eFluor 780 (eBioscience), otherwise DAPI (4,6-diamidino-2-phenylindole; Invitrogen, Carlsbad, CA) was used. EdU was detected applying a Click-it Alexa 647 Kit (Life Technologies, Carlsbad, CA) according to the manufacturer's protocol. Staining of antigen-specific PCs was performed by OVA coupled to Pacific Blue (DRFZ) or Alexa 488 (Invitrogen) after fixation and permeabilization of the cells. Data were acquired on a BD FACS Canto II cytometer and analyzed using the FlowJo software (Treestar, Ashland, OR). Total cell numbers were calculated based on population frequencies measured in defined volumes by a MACSQuant analyzer (Miltenyi Biotec, Bergisch Gladbach, Germany). For the bone marrow, a correction factor was used as previously described. ${ }^{54}$

Quantification of ASCs by ELISPOT. MultiScreenHTS plates (Millipore, Billerica, MA) were pretreated with $35 \%$ ethanol, washed with PBS and coated overnight at $4{ }^{\circ} \mathrm{C}$ with $20 \mu \mathrm{g} \mathrm{ml}^{-1}$ of CT or OVA in PBS. For detection of microbial components, content of the small intestine from littermates of the mice to be analyzed was cultured for 2 days on MacConkey and Columbia blood agar plates (Henry Schein, Melville, NY). Bacterial lysates were prepared by lysis with $10 \mathrm{~mm}$ Tris$\mathrm{HCl}, \mathrm{pH} 8.0,0.1 \mathrm{M} \mathrm{NaCl}, 1 \mathrm{~mm}$ EDTA, 5\% Triton X-100, and $10 \mathrm{mg} \mathrm{ml}^{-1}$ Lysozyme (Sigma-Aldrich, St Louis, MO) at $37^{\circ} \mathrm{C}$ for $30 \mathrm{~min}$ and used for coating of ELISPOT plates. The next day, plates were washed with PBS and blocked with RPMI1640 medium (Life Technologies) supplemented with $10 \%$ fetal calf serum (FCS; SigmaAldrich) at $37^{\circ} \mathrm{C}$ for $2 \mathrm{~h}$. Single cell suspensions were washed and resuspended in RPMI/10\% FCS, pipetted onto the plates, and allowed to secrete for $2 \mathrm{~h}$ at $37^{\circ} \mathrm{C}$ in an incubator with $95 \% \mathrm{O}_{2}$ and $5 \% \mathrm{CO}_{2}$. Plates were washed with PBS/0.01\% Tween (Sigma-Aldrich). Secreted antibodies were detected using biotinylated polyclonal goat antimouse IgM/IgA and IgG antibodies (Southern Biotech), and plates were washed and incubated with streptavidin coupled to alkaline phosphatase (SA-AP, Sigma). After washing, $25 \mu$ l of the substrate $\mathrm{BCIP} / \mathrm{NBT}$ per well was added. The color reaction was stopped with tap water after $3 \mathrm{~min}$, and plates were allowed to dry for $12 \mathrm{~h}$ before they were photographed in an AID ELISPOT Reader (Strassberg, Germany) and analyzed by the ImmunoSpot software (Shaker Heights, $\mathrm{OH})$.

Detection of serum antibodies by enzyme-linked immunosorbent assay. Flat-bottom, high-binding plates (Corning Costar, Tewksbury, MA) were coated with $50 \mu \mathrm{lOVA}\left(20 \mu \mathrm{g} \mathrm{ml}^{-1}\right)$ in PBS overnight, washed, and blocked with PBS $/ 3 \%$ BSA for at $37^{\circ} \mathrm{C}$ for $1 \mathrm{~h}$. Samples in dilution rows were incubated overnight at $4{ }^{\circ} \mathrm{C}$ and washed off with PBS/BSA before adding the same detection antibodies that were used in the ELISPOT experiments $\left(24 \mathrm{~h}\right.$ at $\left.4{ }^{\circ} \mathrm{C}\right)$. After another washing step, $50 \mu \mathrm{l} \mathrm{SA}-\mathrm{AP}$ was added to each well (20 min, room temperature). The substrates ALP1 and ALP2 (Roche, Rotkreuz, Switzerland/Hitachi Cobas, Rotkreuz, Switzerland) were added, and plates were read at $405 \mathrm{~nm}$ on a Spectra Max Plus (Molecular Devices, Sunnyvale, CA). Data were analyzed using SoftMax Pro.

Immunofluorescence histology. Intestines were cleaned, embedded in OCT medium, and snap-frozen in 2-methylbutane in a beaker surrounded by a mix of dry ice and $95 \%$ ethanol. In all, $10-\mu \mathrm{m}$ sections were cut in a Microm HM560 (Thermo Scientific, Waltham, MA) cryostat and transferred onto SuperFrost Plus slides (Thermo Scientific, Waltham, MA). Sections were fixed in ice cold acetone for $10 \mathrm{~min}$, dried, and stored at $-80^{\circ} \mathrm{C}$. Femora were fixed for $6 \mathrm{~h}$ in $4 \%$ paraformaldehyde (Science Services, Munich, Germany) and cryoprotected by sequential incubations in 10, 20, and 30\% sucrose. Freezing and sectioning of whole femur bones was performed according to Kawamoto's method. ${ }^{55}$ Sections were stained in PBS/3\% BSA $/ 0.1 \%$ Tween (intestine) or PBS/5\% FCS $/ 0.1 \%$ Tween (bone marrow) for $1 \mathrm{~h}$ after blocking with $10 \%$ rat serum for $20 \mathrm{~min}$. The following antibodies were used: monoclonal rat anti-mouse kappa light chain FITC (clone 187.1, DRFZ), polyclonal biotinylated goat anti-mouse IgA (Southern Biotech) using streptavidin-Pacific Blue (Invitrogen, coupled in DRFZ), and monoclonal rat anti-mouse IgA (clone, 11-44-2, Southern Biotech) coupled in-house to Pacific Blue. Detection of OVA-specific PCs was performed by staining tissue sections with fluorescently coupled OVA as described above for flow cytometry. EdU incorporation was detected using the EdU Alexa 647 Imaging Kit (Life Technologies) according to the manufacturer's protocol. Nuclei were stained with DAPI or Sytox Green (Invitrogen) for 5-10 min prior to embedding the sections in Prolong Gold medium (Life Technologies) and adding the cover glass. Sections were analyzed by confocal microscopy on a Zeiss LSM710 with ZEN2011 software (Oberkochen, Germany).

Statistical analysis. The absolute cell numbers differences were tested for the statistical significance with two-tailed Mann-Whitney $U$-test using the GraphPad Prism Software, La Jolla, CA.

SUPPLEMENTARY MATERIAL is linked to the online version of the paper at http://www.nature.com/mi

\section{ACKNOWLEDGMENTS}

We thank Sabine Gruczek, Patrick Thiemann and Manuela Ohde for assistance with animal care. We are grateful to Toralf Kaiser and Jenny Kirsch from the DRFZ flow cytometry core facility for help with cell sorting. We thank Robert Günther, Peggy Mex as well as the DRFZ lab managers (Tuula Geske, Heidi Schliemann and Heidi Hecker-Kia and Anette Peddinghaus) for excellent technical assistance. We thank Andreas Radbruch, Bimba Hoyer, Jakob Zimmermann, Claudia Berek, and Van Trung Chu for helpful 
discussions and Randy Lindquist for critical reading of the manuscript. We are grateful to J. and N. Lee, Mayo Clinic, Scottsdale, Arizona, USA for providing us with MBP-specific antibodies. This work was supported by DFG HA5354/4-1, SFB633/TPA15, TRR130/P17 and FOR2165/HA5354/6-1 to A.E.H. M.K. was supported by an IMMUCO integrated research training scholarship within SFB633, and D.L. is a member of the Leibniz Graduate School for Rheumatology (LGRh).

\section{DISCLOSURE}

The authors declared no conflict of interest.

c) 2016 Society for Mucosal Immunology

\section{REFERENCES}

1. Hauser, A.E. et al. Chemotactic responsiveness toward ligands for CXCR3 and CXCR4 is regulated on plasma blasts during the time course of a memory immune response. J. Immunol. 169, 1277-1282 (2002).

2. Hargreaves, D.C. et al. A coordinated change in chemokine responsiveness guides plasma cell movements. J. Exp. Med. 194, 45-56 (2001).

3. Cassese, G. et al. Plasma cell survival is mediated by synergistic effects of cytokines and adhesion-dependent signals. J. Immunol. 171, 1684-1690 (2003).

4. Chu, V.T. et al. Eosinophils are required for the maintenance of plasma cells in the bone marrow. Nat. Immunol. 12, 151-159 (2011).

5. Nagasawa, T. Microenvironmental niches in the bone marrow required for B-cell development. Nat. Rev. Immunol. 6, 107-116 (2006).

6. Radbruch, A. et al. Competence and competition: the challenge of becoming a long-lived plasma cell. Nat. Rev. Immunol. 6, 741-750 (2006).

7. DiLillo, D.J. et al. Maintenance of long-lived plasma cells and serological memory despite mature and memory B cell depletion during CD20 immunotherapy in mice. J. Immunol. 180, 361-371 (2008).

8. Mei, H.E. etal. Blood-borne human plasma cells in steady-state are derived from mucosal immune responses. Blood 113, 2461-2469 (2008).

9. Cerutti, A. \& Rescigno, M. The biology of intestinal immunoglobulin A responses. Immunity 28, 740-750 (2008).

10. Hammerschmidt, S.I. et al. Retinoic acid induces homing of protective T and $\mathrm{B}$ cells to the gut after subcutaneous immunization in mice. J. Clin. Invest. 121, 3051-3061 (2011).

11. Quiding-Jarbrink, M. et al. Differential expression of tissue-specific adhesion molecules on human circulating antibody-forming cells after systemic, enteric, and nasal immunizations. A molecular basis for the compartmentalization of effector B cell responses. J. Clin. Invest. 99, 1281-1286 (1997).

12. Fagarasan, S., Kinoshita, K., Muramatsu, M., Ikuta, K. \& Honjo, T. In situ class switching and differentiation to IgA-producing cells in the gut lamina propria. Nature 413, 639-643 (2001).

13. Youngman, K.R., Franco, M.A., Kuklin, N.A., Rott, L.S., Butcher, E.C. \& Greenberg, H.B. Correlation of tissue distribution, developmental phenotype, and intestinal homing receptor expression of antigen-specific $B$ cells during the murine anti-rotavirus immune response. J. Immunol. 168, 2173-2181 (2002).

14. Kamata, T. et al. Increased frequency of surface IgA-positive plasma cells in the intestinal lamina propria and decreased IgA excretion in hyper IgA (HIGA) mice, a murine model of IgA nephropathy with hyperserum IgA. J. Immunol. 165, 1387-1394 (2000).

15. Mattioli, C.A. \& Tomasi, T.B. Jr. The life span of IgA plasma cells from the mouse intestine. J. Exp. Med. 138, 452-460 (1973).

16. Mesin, L., Di Niro, R., Thompson, K.M., Lundin, K.E. \& Sollid, L.M. Longlived plasma cells from human small intestine biopsies secrete immunoglobulins for many weeks in vitro. J. Immunol. 187, 2867-2874 (2011).

17. Hapfelmeier, S. et al. Reversible microbial colonization of germ-free mice reveals the dynamics of IgA immune responses. Science 328, 1705-1709 (2010).

18. Manz, R.A., Thiel, A. \& Radbruch, A. Lifetime of plasma cells in the bone marrow. Nature 388, 133-134 (1997).

19. Elson, C.O. \& Ealding, W. Generalized systemic and mucosal immunity in mice after mucosal stimulation with cholera toxin. J. Immunol. 132, 2736-2741 (1984).
20. Lycke, N. \& Holmgren, J. Strong adjuvant properties of cholera toxin on gut mucosal immune responses to orally presented antigens. Immunology 59, 301-308 (1986).

21. Vajdy, M. \& Lycke, N.Y. Cholera toxin adjuvant promotes long-term immunological memory in the gut mucosa to unrelated immunogens after oral immunization. Immunology 75, 488-492 (1992).

22. Salic, A. \& Mitchison, T.J. A chemical method for fast and sensitive detection of DNA synthesis in vivo. Proc. Natl. Acad. Sci. USA 105, 2415-2420 (2008).

23. Rothenberg, M.E. \& Hogan, S.P. The eosinophil. Annu. Rev. Immunol. 24, 147-174 (2006).

24. Chu, V.T. et al. Eosinophils promote generation and maintenance of immunoglobulin-A-expressing plasma cells and contribute to gut immune homeostasis. Immunity 40, 582-593 (2014).

25. Odendahl, M. et al. Generation of migratory antigen-specific plasma blasts and mobilization of resident plasma cells in a secondary immune response. Blood 105, 1614-1621 (2005).

26. Macpherson, A.J., Geuking, M.B., Slack, E., Hapfelmeier, S. \& McCoy, K.D. The habitat, double life, citizenship, and forgetfulness of IgA. Immunol. Rev. 245, 132-146 (2012).

27. Puga, I. et al. B cell-helper neutrophils stimulate the diversification and production of immunoglobulin in the marginal zone of the spleen. Nat. Immunol. 13, 170-180 (2012).

28. Haas, A. et al. Systemic antibody responses to gut commensal bacteria during chronic HIV-1 infection. Gut 60, 1506-1519 (2011).

29. Zimmermann, K., Haas, A. \& Oxenius, A. Systemic antibody responses to gut microbes in health and disease. Gut Microbes 3, 42-47 (2012).

30. Slifka, M.K., Antia, R., Whitmire, J.K. \& Ahmed, R. Humoral immunity due to long-lived plasma cells. Immunity 8, 363-372 (1998).

31. Hiepe, F., Dorner, T., Hauser, A.E., Hoyer, B.F., Mei, H. \& Radbruch, A. Long-lived autoreactive plasma cells drive persistent autoimmune inflammation. Nat. Rev. Rheumatol. 7, 170-178 (2011).

32. Lycke, N. \& Bemark, M. Mucosal adjuvants and long-term memory development with special focus on CTA1-DD and other ADP-ribosylating toxins. Mucosal Immunol. 3, 556-566 (2010).

33. Casola, S. \& Rajewsky, K. B cell recruitment and selection in mouse GALT germinal centers. Curr. Top. Microbiol. Immunol. 308, 155-171 (2006).

34. Roth, K., Oehme, L., Zehentmeier, S., Zhang, Y., Niesner, R. \& Hauser, A.E. Tracking plasma cell differentiation and survival. Cytometry A 85, 15-24 (2014).

35. Hofer, T. et al. Adaptation of humoral memory. Immunol. Rev. 211, 295-302 (2006).

36. Peperzak, V. et al. Mcl-1 is essential for the survival of plasma cells. Nat. Immunol. 14, 290-297 (2013).

37. Zehentmeier, S. et al. Static and dynamic components synergize to form a stable survival niche for bone marrow plasma cells. Eur. J. Immunol. 44, 2306-2317 (2014).

38. He, B. et al. Intestinal bacteria trigger T cell-independent immunoglobulin A(2) class switching by inducing epithelial-cell secretion of the cytokine APRIL. Immunity 26, 812-826 (2007).

39. Heremans, J.F. Immunoglobulin A. In The Antigens vol. 2 (Sela, M., ed) 365-522 (Academic Press Inc, USA, 1974).

40. Matsui, H., Isshiki, Y., Eguchi, M., Ogawa, Y. \& Shimoji, Y. Evaluation of the live vaccine efficacy of virulence plasmid-cured, and phoP- or aroAdeficient Salmonella enterica serovar Typhimurium in mice. J. Vet. Med. Sci. 77, 181-186 (2015).

41. Macpherson, A.J., Gatto, D., Sainsbury, E., Harriman, G.R., Hengartner, H. \& Zinkernagel, R.M. A primitive Tcell-independent mechanism of intestinal mucosal IgA responses to commensal bacteria. Science 288, 2222-2226 (2000).

42. Konrad, A., Cong, Y., Duck, W., Borlaza, R. \& Elson, C.O. Tight mucosal compartmentation of the murine immune response to antigens of the enteric microbiota. Gastroenterology 130, 2050-2059 (2006).

43. Natividad, J.M. et al. Host responses to intestinal microbial antigens in gluten-sensitive mice. PLoS One 4, e6472 (2009).

44. Chow, J., Tang, H. \& Mazmanian, S.K. Pathobionts of the gastrointestinal microbiota and inflammatory disease. Curr. Opin. Immunol. 23, 473-480 (2011).

45. Wu, H.J. et al. Gut-residing segmented filamentous bacteria drive autoimmune arthritis via T helper 17 cells. Immunity 32, 815-827 (2010). 
46. Bernasconi, N.L., Traggiai, E. \& Lanzavecchia, A. Maintenance of serological memory by polyclonal activation of human memory B cells. Science 298, 2199-2202 (2002).

47. Bernasconi, N.L., Onai, N. \& Lanzavecchia, A. A role for Toll-like receptors in acquired immunity: up-regulation of TLR9 by BCR triggering in naive $B$ cells and constitutive expression in memory B cells. Blood 101, 4500-4504 (2003).

48. Lindner, C. et al. Age, microbiota, and T cells shape diverse individual IgA repertoires in the intestine. J. Exp. Med. 209, 365-377 (2012).

49. Bemark, M., Bergqvist, P., Stensson, A., Holmberg, A., Mattsson, J. \& Lycke, N.Y. A unique role of the cholera toxin A1-DD adjuvant for long-term plasma and memory B cell development. J. Immunol. 186, 1399-1410 (2011).

50. Spencer, J., Klavinskis, L.S. \& Fraser, L.D. The human intestinal IgA response; burning questions. Front. Immunol. 3, 108 (2012).
51. Pabst, O. New concepts in the generation and functions of IgA. Nat. Rev. Immunol. 12, 821-832 (2012).

52. Bakema, J.E. \& van Egmond, M. The human immunoglobulin A Fc receptor FcalphaRl: a multifaceted regulator of mucosal immunity. Mucosal Immunol. 4, 612-624 (2011).

53. Mestecky, J., Russell, M.W. \& Elson, C.O. Intestinal IgA: novel views on its function in the defence of the largest mucosal surface. Gut 44, 2-5 (1999).

54. Chervenick, P.A., Boggs, D.R., Marsh, J.C., Cartwright, G.E. \& Wintrobe, M.M. Quantitative studies of blood and bone marrow neutrophils in normal mice. Am. J. Physiol. 215, 353-360 (1968).

55. Kawamoto, T. Use of a new adhesive film for the preparation of multipurpose fresh-frozen sections from hard tissues, whole-animals, insects and plants. Arch. Histol. Cytol. 66, 123-143 (2003). 